\title{
MHD Free Convection Flow Across an Inclined Porous Plate in the Presence of Heat Source, Soret Effect, and Chemical Reaction Affected by Viscous Dissipation Ohmic Heating
}

\author{
Bijoy Krishna Taid ${ }^{1, *(\mathbb{D})}$, Nazibuddin Ahmed ${ }^{1(\mathbb{D})}$ \\ 1 Department of Mathematics, Gauhati University, Guwahati-781014, Assam, India; bijoytaid@gmail.com_(B.K.T); \\ nazib@gauhati.ac.in_(N.A.); \\ * Correspondence: bijoytaid@gmail.com (B.K.T);
}

Scopus Author ID 9636158600

Received: 15.06.2021; Revised: 20.09.2021; Accepted: 25.09.2021; Published: 9.11.2021

\begin{abstract}
This work studies the steady two-dimensional MHD free convection flow past an inclined porous plate embedded in the porous medium in the presence of heat source, Soret effect, and chemical reaction. The non-dimensional governing equations are solved by the perturbation technique. The Rosseland approximation is utilized to describe the radiative heat flux in the energy equation. The effect of magnetic parameter, heat source parameter, radiation parameter, Grashof number, modified Grashof $\mathrm{i}$ number, Schmidt number, Prandtl number, porosity parameter, Soreti number, and chemical reaction on velocity, temperature, concentration profiles, skin friction, Nusselt number, and Sherwood number are mainly focussed in discussion with the help of graphs. It is seen that velocity, concentration, and skin friction fall with the increasing value of chemical reaction. Further, temperature, Nusselt number, and Sherwood number increase with the increasing value of chemical reaction.
\end{abstract}

Keywords: inclined plate; Soret effect; chemical reaction; porous medium; heat source.

(C) 2021 by the authors. This article is an open-access article distributed under the terms and conditions of the Creative Commons Attribution (CC BY) license (https://creativecommons.org/licenses/by/4.0/).

\section{Nomenclature:}

$\vec{q}$ Fluid Velocity;

$B_{0}$ Strength of magnetic field;

$v_{0}$ Suction velocity;

$u_{0}$ velocity of plate;

$C_{p}$ Specific heat at const. pressure;

$q_{r}$ Radioactive heat flux;

$M$ Magnetic parameter;

Pr Prandtl number;

E Eckert number;

Sr Soret number;

$D_{M}$ Mass dissusivity;

$K_{T}$ Thermal diffusion ratio;

$R$ Chemical reaction;
$T$ Fluid temperature;

$T_{m}$ Mean fluid temperature;

$T_{w}$ wall temperature;

$T_{\infty}$ Fluid temperature far away from the plate;

C Species concentration;

$C_{w}$ Wall concentration;

$C_{\infty}$ Concentration far away from the plate;

u Dimensionless velocity;

$\rho$ Fluid density;

$v$ Kinematic viscosity;

$\sigma$ Electrical conductivity;

$\kappa$ Thermal conductivity;

$\mu$ Coefficient of viscosity;

$\beta$ Volumetric coefficient of thermal expansion; 
$F$ Radiation parameter;

$S$ heat source;

Gr Grashof number;

Gm Modified Grashof number;

$K$ Porosity parameter;

Sc Schmidt number; $\beta_{c}$ Volumetric coefficient of thermal expansion

with concentration;

$\theta$ Dimensionless temperature;

$\phi$ Dimensionless concentration;

\section{Introduction}

The study of magnetohydrodynamics nowadays has sparked a lot of interest and concern due to its applications in petroleum industries, gas turbines, plasma physics, food processing industries, geothermal energy, nuclear power plants, cooling of nuclear reactors, and lubrication industries, etc. It is also utilized in labs for chemical, medicinal, and biological research. Alfven [1] was the first to use the concept of magnetohydrodynamics. Several significant authors, including Cowling [2], Ferraro and Plumpton [3], and Crammer and Pai [4], contributed to the current form of MHD. On the other hand, several academics have researched MHD flows. In the presence of an induced magnetic field, Singh and Singh [5] investigated the influence of MHD on heat and mass transfer in a viscous fluid. They [6] also demonstrated MHD free convection and mass transfer flow through a flat plate. In the presence of viscous dissipation, MHD natural convection flow heat and mass transfer in a moving vertical plate with the convective condition were analyzed by Shateyi [7]. In the presence of a constant heat source, Lawal and Jibril [8] examined the impact of relative motion of a magnetic field on unsteady MHD natural convection flow. Ramakrishna et al. [9] evaluated the effect of an aligned magnetic field on an unstable natural convective flow past an inclined vertical plate. Ilias et al. [10] studied the flow and heat transfer of magnetic nanofluids past an inclined plate in an unsteady aligned MHD boundary layer. MHD mixed convection flow of a maxwell nanofluid in the presence of varying thermal conductivity, Dufour, and Soret effects was studied analytically by Jawad et al. [11]. In a magnetohydrodynamic flow of nanofluid, Masood et al. [12] described viscous dissipation. The effects of suction/injection, the permeability of porous materials, and viscous dissipation on an electrically conducting incompressible fluid passing through a vertical plate were studied theoretically by Ajibade $e t$ al. [13]. Thermal radiation and chemical reactions have an impact on magnetohydrodynamic nanofluid flow past a stretching vertical surface explored by Devi et al.[14]. Ganga et al. [15] looked at how an inclined magnetic field affected entropy formation in a nanofluid over a stretching sheet with partial slip and non-linear thermal radiation. Dar [16] studied the impact of thermal radiation, temperature jumps, and an angled magnetic field on peristaltic blood flow transfer in an asymmetric channel with varying viscosity and heat absorption/generation. Selvarasu and Balamurugan [17] investigate the effects of viscous dissipation, ohmic heating, and chemical reaction on MHD free convection flow past an inclined porous plate with a heat source. Parashar and Ahmed [18] study the effects of Hall current, ion slip current, and chemical reaction on unsteady MHD convective flow of liquid lead through an impulsively started semi-infinite vertical porous plate in the presence of a uniform magnetic field. In the presence of radiation and Soret effects, Ahmed [19] investigated the influence of transient magnetohydrodynamics free convection flow from an impulsively begun infinite vertical plate. Jena et al. [20] published a paper on the effect of chemical reactions on magnetohydrodynamics viscoelastic fluid flow over a vertical stretching sheet with a heat source/sink. Gurram et al. 
[21] examine the influence of a chemical reaction on the magnetohydrodynamics Casson fluid flow along an inclined surface with radiation. In the presence of hall current, Raju [22] examined the role of a chemical reaction and associated buoyancy effects on an unstable MHD mixed convective flow along with an infinite vertical porous plate. Rashidi et al. [23] analyzed heat and mass transfer in a constant MHD viscoelastic fluid flow over a vertical stretching sheet in the presence of Soret and Dufour effects. Venkateswarlu et al. [24] investigated in the presence of heat generation the radiation effects of mass transfer on non-linear MHD boundary layer flow of a viscous incompressible fluid through a non-linear porous stretching surface in a porous medium. Bilal and Nazeer numerically analyzed the non-Newtonian flow across a stratified stretching/shrinking inclined sheet with an aligned magnetic field and non-linear convection [25]. Usharani and Selvaraj [26] investigated the influence of a magnetohydrodynamics stream passing through an exponentially inclined vertical plate of firstorder chemical reaction with variable mass diffusion and thermal radiation. Megaraju and Shekar [27] evaluated the transient magnetohydrodynamics current of an exponentially accelerated isothermal vertical plate, considering the effects of hall current and chemical reactions. In the presence of a chemical reaction, Reddy and Reddy [28] investigated the effects of viscous dissipation on free convection MHD flow through a porous medium across an exponentially stretching surface. Oyekunle and Agunbiade [29] examined the effects of several physical parameters on unsteady MHD slip flow over a permeable vertical plate, including thermal radiation, viscous dissipation, and inclined magnetic field. With chemical reaction, arbitrary shear stress, and exponential heating, Sehra et al. [30] studied convective heat mass transfer and MHD flow over a vertical plate.

\section{Mathematical analysis}

We consider the two-dimensional electrically conducting viscous incompressible flow with chemical reaction and Soret effect along with a semi-infinite inclined porous plate at an angle $\alpha$ to the vertical direction embedded in a porous medium. The temperature and concentration at stretching surface are $T_{w}$ and $C_{w}$, while these values at free stream are $T_{\infty}$ and $C_{\infty}$ respectively. The physical coordinates- $\left(x^{\prime}, y^{\prime}\right)$ are chosen such that $x^{\prime}$-axis taken along the direction of motion and $y^{\prime}$-axis taken to be normal. The flow is governed by the following system of equations using the standard Boussinesq approximations.

$$
\begin{aligned}
& \frac{\partial v^{\prime}}{\partial y^{\prime}}=0 \\
& \rho v^{\prime} \frac{\partial u^{\prime}}{\partial y^{\prime}}=\mu \frac{\partial^{2} u^{\prime}}{\partial y^{\prime 2}}-\sigma B_{0}{ }^{2} u^{\prime}+\rho g \beta\left(T-T_{\infty}\right) \cos \alpha+\rho g \beta_{c}\left(C-C_{\infty}\right) \cos \alpha-\frac{\mu u^{\prime}}{K^{*}} \\
& \rho C_{p} v^{\prime} \frac{\partial T}{\partial y^{\prime}}=\kappa \frac{\partial^{2} T}{\partial y^{\prime 2}}+Q\left(T-T_{\infty}\right)-\frac{\partial q_{r}}{\partial y^{\prime}}+\mu\left(\frac{\partial u^{\prime}}{\partial y^{\prime}}\right)^{2}+\sigma B_{0}{ }^{2} u^{\prime 2} \\
& v^{\prime} \frac{\partial C}{\partial y^{\prime}}=D_{M} \frac{\partial^{2} C}{\partial y^{\prime 2}}+\bar{K}\left(C_{\infty}-C\right)+\frac{D_{M} K_{T}}{T_{m}} \frac{\partial^{2} T}{\partial y^{\prime 2}}
\end{aligned}
$$

The corresponding boundary conditions

at $y^{\prime}=0 ; u^{\prime}=0, T=T_{w}, C=C_{w}$

as $y^{\prime} \rightarrow \infty ; u^{\prime} \rightarrow 0, T \rightarrow T_{\infty}, C \rightarrow C_{\infty}$ 
Consider the fluid which is optically thin with a relatively flow density and radioactive heat flux is given by $\frac{\partial q_{r}}{\partial y^{\prime}}=4\left(T \rightarrow T_{\infty}\right) I$, Where $I$ is the absorption coefficient at the plate.

From (1.1) we have $v^{\prime}=-v_{0}$, where negative sign indicates that suction velocity is directed towards the plate.

Introduce non-dimensional quantities to normalize the governing equations:

$y=\frac{v_{0} y^{\prime}}{v}, \quad u=\frac{u^{\prime}}{v_{0}}, \quad M=\frac{\sigma B_{0}{ }^{2} v}{\rho v_{0}{ }^{2}}, \quad G r=\frac{\rho \beta v\left(T_{w}-T_{\infty}\right)}{v_{0}{ }^{3}}, \quad G m=\frac{\rho \beta_{c} v\left(C_{w}-C_{\infty}\right)}{v_{0}{ }^{3}}, K=\frac{K^{*} v_{0}{ }^{2}}{v^{2}}$,

$\theta=\frac{T-T_{\infty}}{T_{w}-T_{\infty}}, \quad \phi=\frac{C-C_{\infty}}{C_{w}-C_{\infty}}, \operatorname{Pr}=\frac{\mu C_{p}}{\kappa}, E=\frac{v_{0}{ }^{2}}{C_{p}\left(T_{w}-T_{\infty}\right)}, \quad R=\frac{\bar{K} v}{v_{0}{ }^{2}}, S=\frac{Q v}{\rho C_{p} v_{0}{ }^{2}}$,

$F=\frac{4 v I}{\rho C_{p} v_{0}^{2}}, \quad S c=\frac{v}{D_{M}}, \quad S r=\frac{D_{M} K_{T}\left(T_{w}-T_{\infty}\right)}{v T_{m}\left(C_{w}-C_{\infty}\right)}$.

Substituting (2.3) and dimensionless quantities (3.1) in equation (1.2) - (1.4) we get,

$\frac{\partial^{2} u}{\partial y^{2}}+\frac{\partial u}{\partial y}-\left(M+\frac{1}{K}\right) u=-G r \cos \alpha \theta-G m \cos \alpha \phi$

$\frac{\partial^{2} \theta}{\partial y^{2}}+\operatorname{Pr} \frac{\partial \theta}{\partial y}-\operatorname{Pr}(F-S) \theta=-\operatorname{Pr} E\left(\frac{\partial u}{\partial y}\right)^{2}-\operatorname{Pr} E M u^{2}$

$\frac{\partial^{2} \phi}{\partial y^{2}}+S c \frac{\partial \phi}{\partial y}-R S c \phi=-S r S c \frac{\partial^{2} \theta}{\partial y^{2}}$

Subject to the boundary conditions

at $y=0 ; u=0, \theta=1, \phi=1$

as $y \rightarrow 0 ; u \rightarrow 0, \theta \rightarrow 0, \phi \rightarrow 0$

\section{Method of Solution}

The governing equations (4.1), (4.2), and (4.3) are non-linear, and, in order to obtain a solution, we expand in powers of Eckert number assuming to be small. So that we can express it as a regular perturbation series

$$
\begin{aligned}
& u(y)=u_{0}+\varepsilon u_{1}+o\left(\varepsilon^{2}\right) \\
& \theta(y)=\theta_{0}+\varepsilon \theta_{1}+o\left(\varepsilon^{2}\right) \\
& \phi(y)=\phi_{0}+\varepsilon \phi_{1}+o\left(\varepsilon^{2}\right)
\end{aligned}
$$

Substituting (6.1)-(6.3) in equations (4.1), (4.2), and (4.3) and neglecting higher-order terms of $o\left(\varepsilon^{2}\right)$ and equating the coefficient of similar terms, then we obtained the following zeroth-order and first-order- differential equations.

$$
\begin{aligned}
& u_{0}^{\prime \prime}+u_{0}^{\prime}-\left(M+\frac{1}{K}\right) u_{0}=-G r \cos \alpha \theta_{0}-G m \cos \alpha \phi_{0} \\
& \theta_{0}^{\prime \prime}+\operatorname{Pr} \theta_{0}^{\prime}-\operatorname{Pr}(F-S) \theta_{0}=0 \\
& \phi_{0}^{\prime \prime}+S c \phi_{0}^{\prime}-R S c \phi_{0}=-S r S c \theta_{0}^{\prime \prime} \\
& \text { https://biointerfaceresearch.com/ }
\end{aligned}
$$




$$
\begin{aligned}
& u_{1}^{\prime \prime}+u_{1}^{\prime}-\left(M+\frac{1}{K}\right) u_{1}=-G r \cos \alpha \theta_{1}-G m \cos \alpha \phi_{1}
\end{aligned}
$$

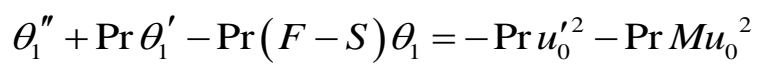

$$
\begin{aligned}
& \phi_{1}^{\prime \prime}+S c \phi_{1}^{\prime}-R S c \phi_{1}=-\operatorname{SrSC} \theta_{1}^{\prime \prime}
\end{aligned}
$$

Boundary conditions (5.1 and (5.2) reduce to the form

$y=0 ; u_{0}=0, u_{1}=0, \theta_{0}=1, \theta_{1}=0, \phi_{0}=1, \phi_{1}=0$

$y \rightarrow 0 ; u_{0}=0, u_{1}=0, \theta_{0}=0, \theta_{1}=0, \phi_{0}=0, \phi_{1}=0$

\section{Solutions of zeroth-order Equations}

The solutions of (7.1) - (7.3) with the boundary conditions (8.1) and (8.2) we get

$$
\begin{aligned}
& u_{0}=A_{6} e^{m_{3} y}+A_{4} e^{m_{2} y}+\left(A_{3}+A_{5}\right) e^{m_{1} y} \\
& \theta_{0}=e^{m_{1} y} \\
& \phi_{0}=A_{1} e^{m_{1} y}+A_{2} e^{m_{2} y}
\end{aligned}
$$

\section{Solutions of first order Equations}

The solutions of (7.4) - (7.6) with the corresponding boundary conditions (8.1) and (8.2) we have,

$$
\begin{aligned}
& u_{1}=A_{36} e^{m_{4} y}+A_{28} e^{m_{1} y}+A_{29} e^{2 m_{3} y}+A_{30} e^{2 m_{1} y}+A_{31} e^{2 m_{2} y}+A_{32} e^{\left(m_{1}+m_{3}\right) y}+A_{33} e^{\left(m_{1}+m_{2}\right) y} \\
& +A_{34} e^{\left(m_{2}+m_{3}\right) y}+A_{35} e^{m_{2} y} \\
& \theta_{1}=A_{9} e^{m_{1} y}+\left(A_{7}+A_{13}\right) e^{2 m_{3} y}+\left(A_{8}+A_{14}\right) e^{2 m_{1} y}+\left(A_{9}+A_{15}\right) e^{2 m_{2} y}+\left(A_{10}+A_{18}\right) e^{\left(m_{1}+m_{3}\right) y} \\
& +\left(A_{11}+A_{17}\right) e^{\left(m_{1}+m_{2}\right) y}+\left(A_{12}+A_{16}\right) e^{\left(m_{2}+m_{3}\right) y} \\
& \phi_{1}=A_{27} e^{m_{2} y}+A_{20} e^{m_{1} y}+A_{21} e^{2 m_{3} y}+A_{22} e^{2 m_{1} y}+A_{23} e^{2 m_{2} y}+A_{24} e^{\left(m_{1}+m_{3}\right) y} \\
& +A_{25} e^{\left(m_{1}+m_{2}\right) y}+A_{26} e^{\left(m_{2}+m_{3}\right) y}
\end{aligned}
$$

Substituting (9.1) - (9.6) in (6.1) - (6.3) we get the following velocity, temperature and concentration distributions

$$
\begin{aligned}
& u(y)=A_{6} e^{m_{3} y}+A_{4} e^{m_{2} y}+\left(A_{3}+A_{5}\right) e^{m_{1} y}+E\left(A_{36} e^{m_{3} y}+A_{28} e^{m_{1} y}+A_{29} e^{2 m_{3} y}\right. \\
& \left.+A_{30} e^{2 m_{1} y}+A_{31} e^{2 m_{2} y}+A_{32} e^{\left(m_{1}+m_{3}\right) y}+A_{33} e^{\left(m_{1}+m_{2}\right) y}+A_{34} e^{\left(m_{2}+m_{3}\right) y}+A_{35} e^{m_{2} y}\right) \\
& \theta(y)=e^{m_{1} y}+E\left\{A_{9} e^{m_{1} y}+\left(A_{7}+A_{13}\right) e^{2 m_{3} y}+\left(A_{8}+A_{14}\right) e^{2 m_{1} y}+\left(A_{9}+A_{15}\right) e^{2 m_{2} y}\right. \\
& \left.+\left(A_{10}+A_{18}\right) e^{\left(m_{1}+m_{3}\right) y}+\left(A_{11}+A_{17}\right) e^{\left(m_{1}+m_{2}\right) y}+\left(A_{12}+A_{16}\right) e^{\left(m_{2}+m_{3}\right) y}\right\} \\
& \phi(y)=A_{1} e^{m_{1} y}+A_{2} e^{m_{2} y}+E\left\{A_{27} e^{m_{2} y}+A_{20} e^{m_{1} y}+A_{21} e^{2 m_{3} y}+A_{22} e^{2 m_{1} y}\right. \\
& \left.+A_{23} e^{2 m_{2} y}+A_{24} e^{\left(m_{1}+m_{3}\right) y}+A_{25} e^{\left(m_{1}+m_{2}\right) y}+A_{26} e^{\left(m_{2}+m_{3}\right) y}\right\}
\end{aligned}
$$

\section{Skin Friction}

The co-efficient of skin friction is given by

$$
\begin{aligned}
& \left.\tau=-\frac{\partial u}{\partial y}\right]_{y=0} \\
& =-\left[m_{3} A_{6}+m_{2} A_{4}+m_{1}\left(A_{3}+A_{5}\right)+E\left(m_{3} A_{36}+m_{1} A_{28}+2 m_{3} A_{29}+2 m_{1} A_{30}+2 m_{2} A_{31}\right.\right. \\
& \left.\left.+\left(m_{1}+m_{3}\right) A_{32}+\left(m_{1}+m_{2}\right) A_{33}+\left(m_{2}+m_{3}\right) A_{34}+m_{2} A_{35}\right)\right]
\end{aligned}
$$




\section{Nusselt Number}

Rate of heat transfer in terms of Nusselt number is given by

$$
\begin{aligned}
& \left.N u=-\frac{\partial \theta}{\partial y}\right]_{y=0} \\
& =-\left[m_{1}+E\left\{m_{1} A_{9}+2 m_{3}\left(A_{7}+A_{13}\right)+2 m_{1}\left(A_{8}+A_{14}\right)+2 m_{2}\left(A_{9}+A_{15}\right)+\left(m_{1}+m_{3}\right)\left(A_{10}+A_{18}\right)\right.\right. \\
& \left.\left.+\left(m_{1}+m_{2}\right)\left(A_{11}+A_{17}\right)+\left(m_{2}+m_{3}\right)\left(A_{12}+A_{16}\right)\right\}\right]
\end{aligned}
$$

\section{Sherwood number}

Rate of mass transfer in terms of Sherwood number is given by

$$
\begin{aligned}
& \left.S h=-\frac{\partial \phi}{\partial y}\right]_{y=0} \\
& =-\left[m_{1} A_{1}+m_{2} A_{2}+E\left\{m_{2} A_{27}+m_{1} A_{20}+2 m_{3} A_{21}+2 m_{1} A_{22}+2 m_{2} A_{23}+\left(m_{1}+m_{3}\right) A_{24}\right.\right. \\
& \left.\left.+\left(m_{1}+m_{2}\right) A_{25}+\left(m_{2}+m_{3}\right) A_{26}\right\}\right]
\end{aligned}
$$

\section{Results and Discussion}

We investigate the influence of physical parameters on velocity, temperature, concentration profiles, skin friction, Nusselt number, and Sherwood number with the help of graphs. The graphs are drawn by Matlab. The influence of chemical reaction, radiation parameter, magnetic parameter, heat source, magnetic parameter, heat source, modified Grashof number, Grashof number, porosity parameter, and Soret effect on velocity profile are shown in Figures 1-8. From Figures 1-3, we observed that the velocity profiles are reduced with the augmented values of chemical reaction, radiation parameter, and magnetic parameter. That is, from Figure1, the consumption of species drops the fluid velocity to a considerable extend. Figures 4-8 depict that the velocity increases with increasing values of heat source, modified Grashof number, Grashof number, porosity parameter, and Soret effect, respectively.

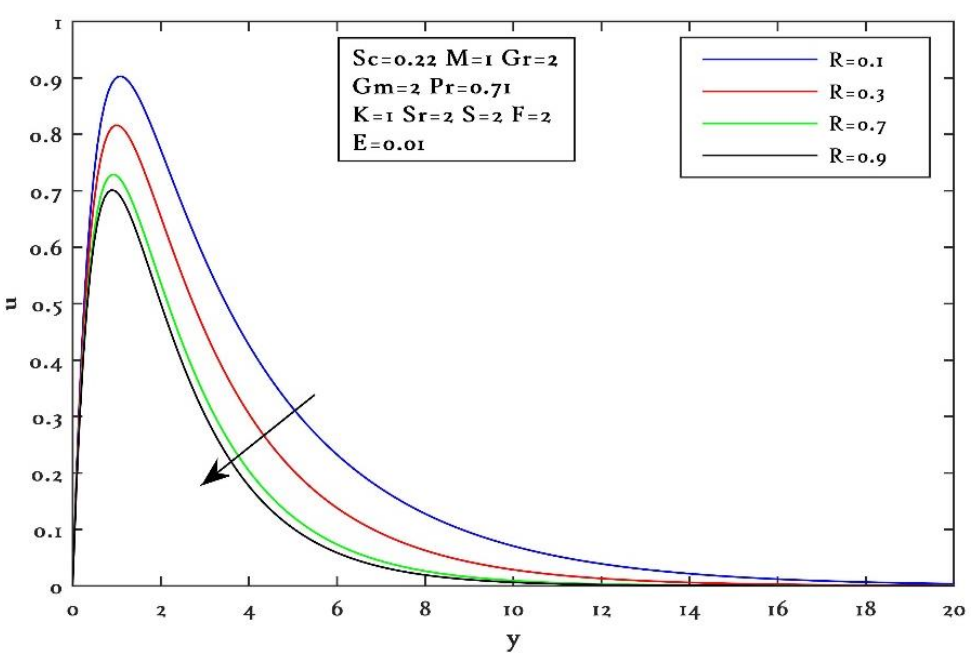

Figure 1. Velocity profile for different values of $\mathrm{R}$ when $\alpha=\frac{\pi}{6}$.

The effect of Grashof number, modified Grashof number, heat source, chemical reaction, radiation parameter, and Prandtl number on temperature is demonstrated in Figures 9- 
14. Figures 9-12 show that the upsurge in the Grashof number, modified Grashof number, heat source, chemical reaction factor increases the temperature. The increasing radiation parameter values and Prandtl number reduce the temperature, as shown in Figures 13 and 14. Therefore, from Figure 14, high thermal diffusivity causes the temperature field to rise to a considerable extend.

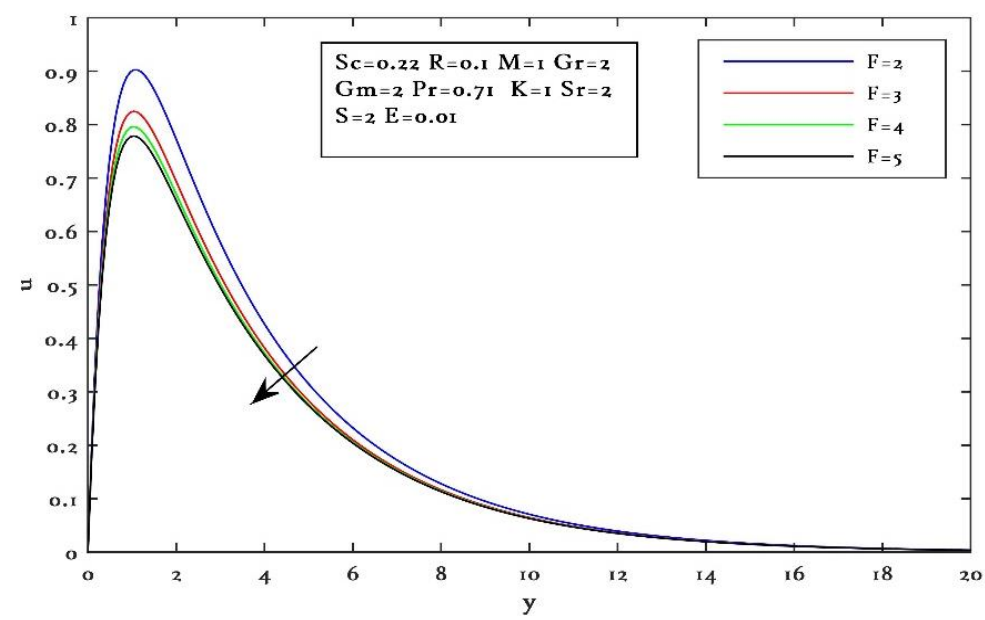

Figure 2. Velocity profile for different values of $\mathrm{F}$ when $\alpha=\frac{\pi}{6}$.
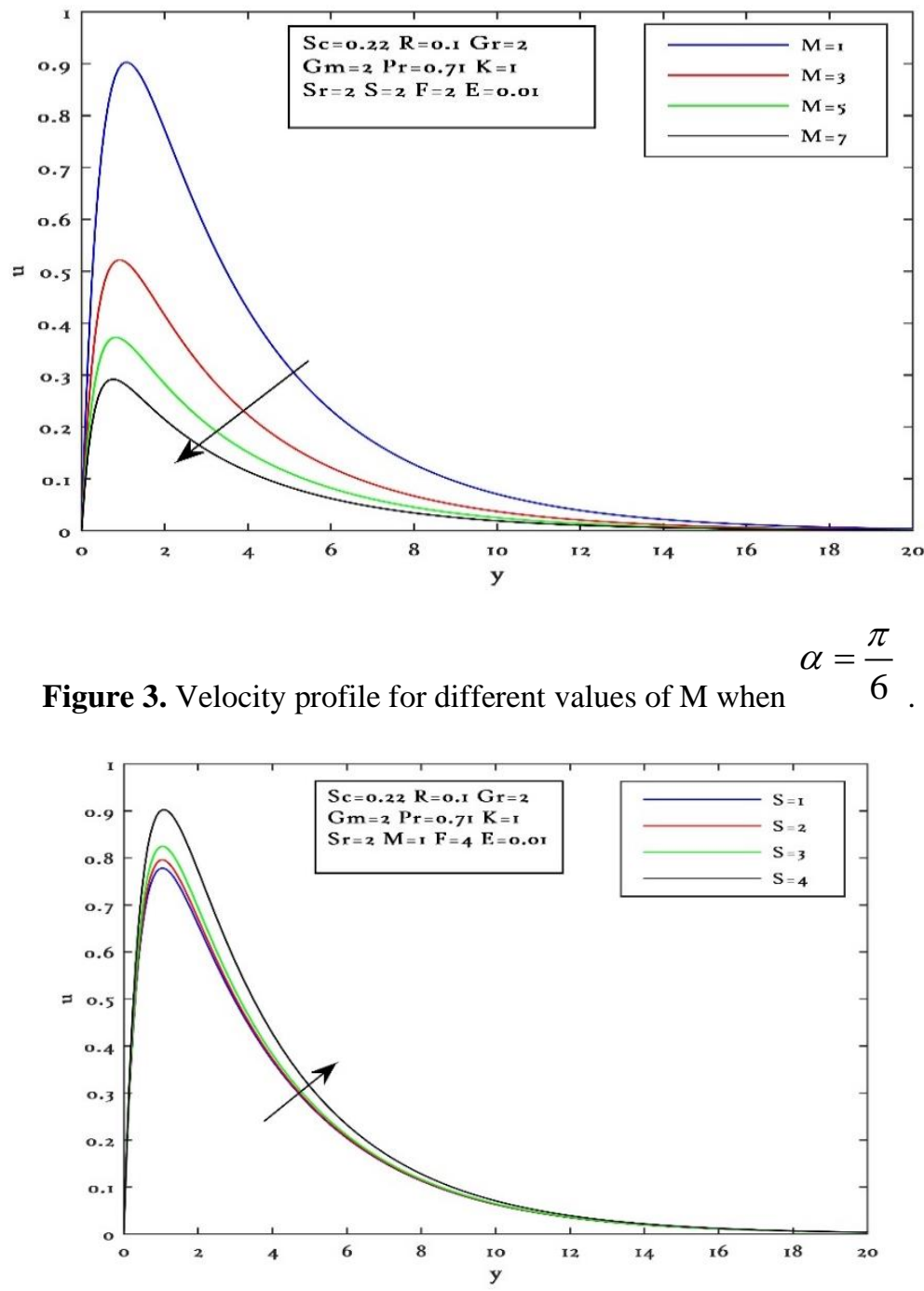

Figure 4. Velocity profile for different values of S when $\alpha=\frac{\pi}{6}$. 


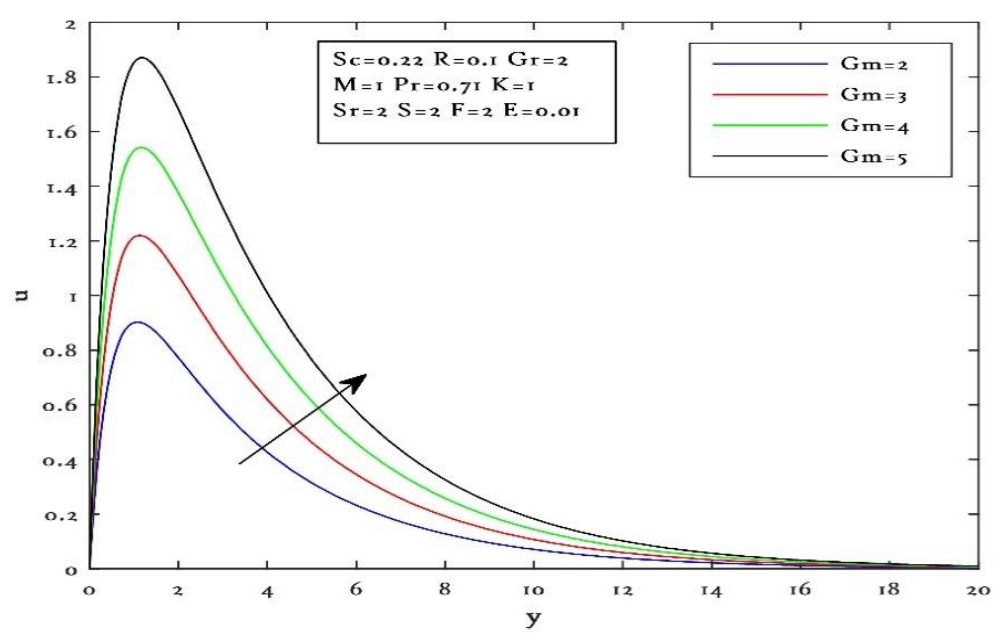

Figure 5. Velocity profile for different values of Gm when $\alpha=\frac{\pi}{6}$.

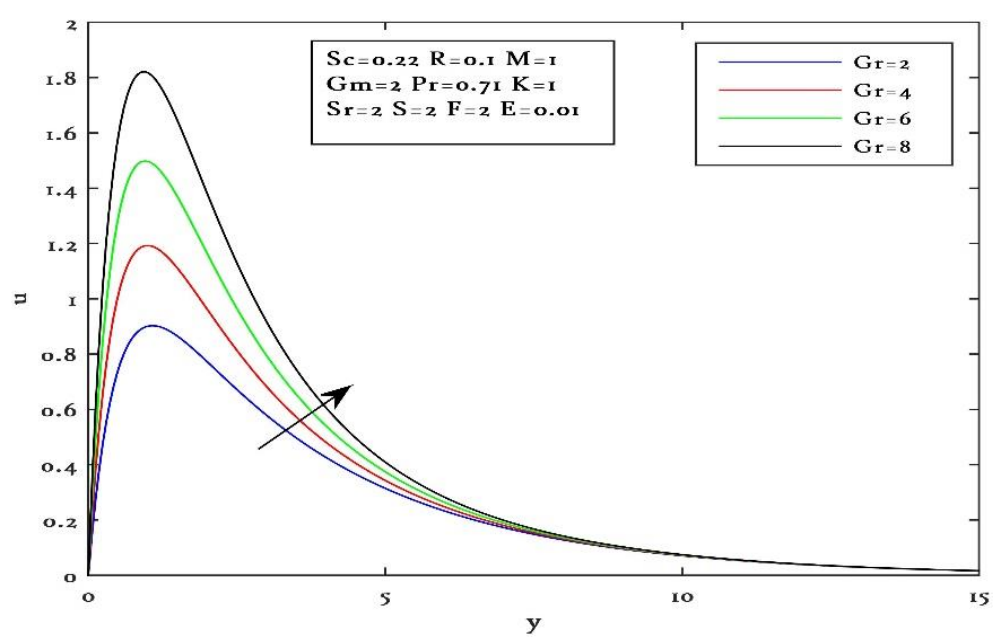

Figure 6. Velocity profile for different values of Gr when $\alpha=\frac{\pi}{6}$.

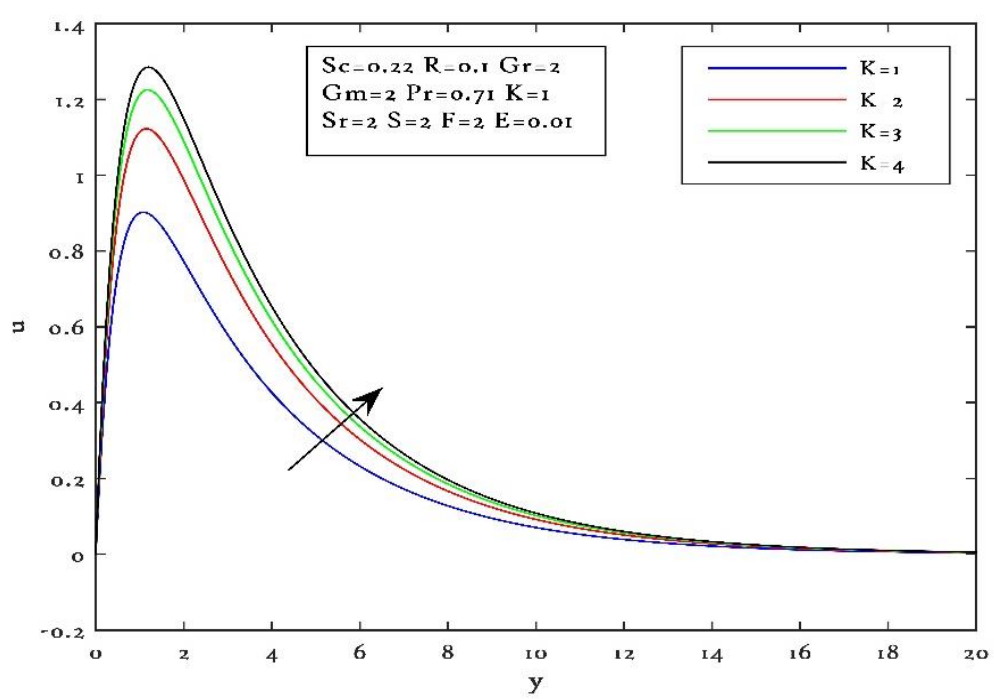

Figure 7. Velocity profile for different values of $\mathrm{K}$ when $\alpha=\frac{\pi}{6}$. 


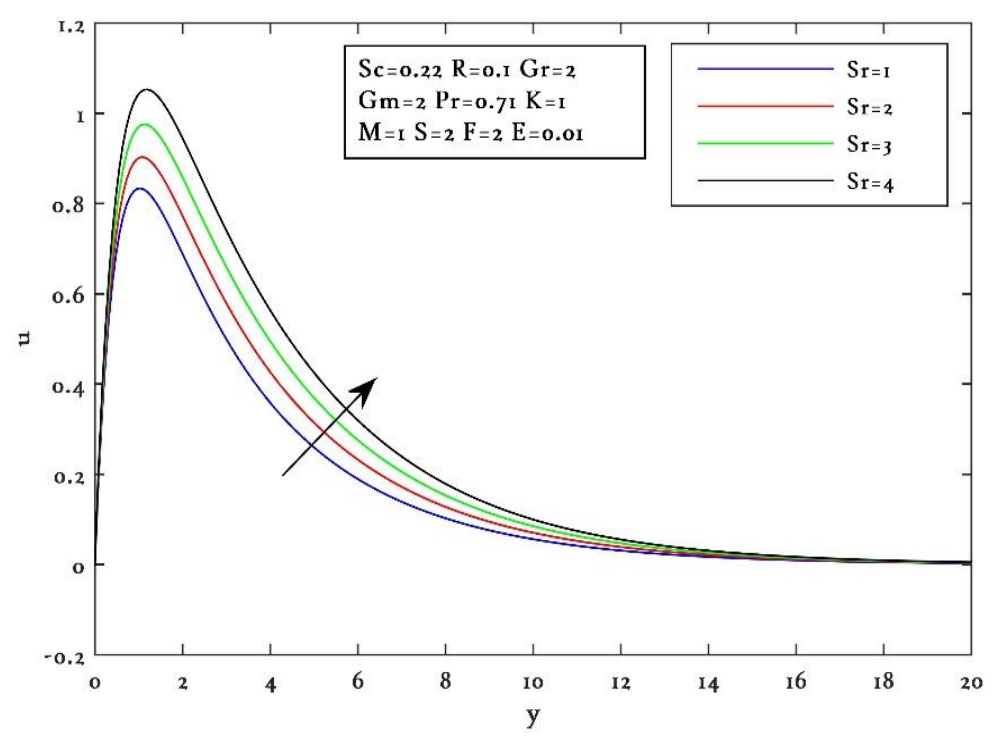

Figure 8. Velocity profile for different values of Sr when $\alpha=\frac{\pi}{6}$.

The influence of chemical reaction and Soret effect on the concentration is shown in Figures 15 and 16. Figure 15 displays that the concentration distribution decreases with growing values of a chemical reaction, and it increases with rising values of Soret number, as shown in Figure 16. Figures 17 and 18 show how skin friction changes when a chemical reaction and porosity parameters change. It is observed that the skin friction falls as the chemical reaction increases. Further, skin friction increases with an increase in porosity parameters. From Figures 19 and 20, it is noticed that the Nusselt number increase with the increasing values of a chemical reaction and Schmidt number. So that Nusselt number or rate of heat transfer falls for increasing mass diffusivity. The behavior of the Sherwood number is presented in Figures 21 and 22. It is clear that the Sherwood number or mass transfer rate increases under the influence of chemical reaction and porosity parameters.

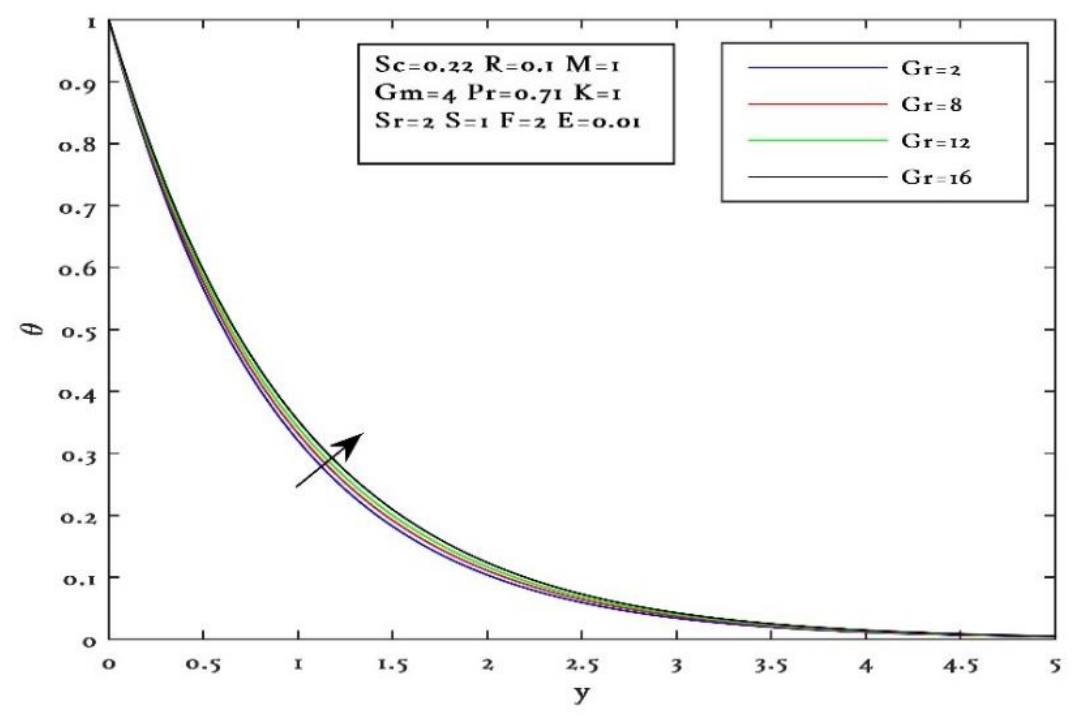

Figure 9. Temperature for different values of Gr when $\alpha=\frac{\pi}{6}$. 


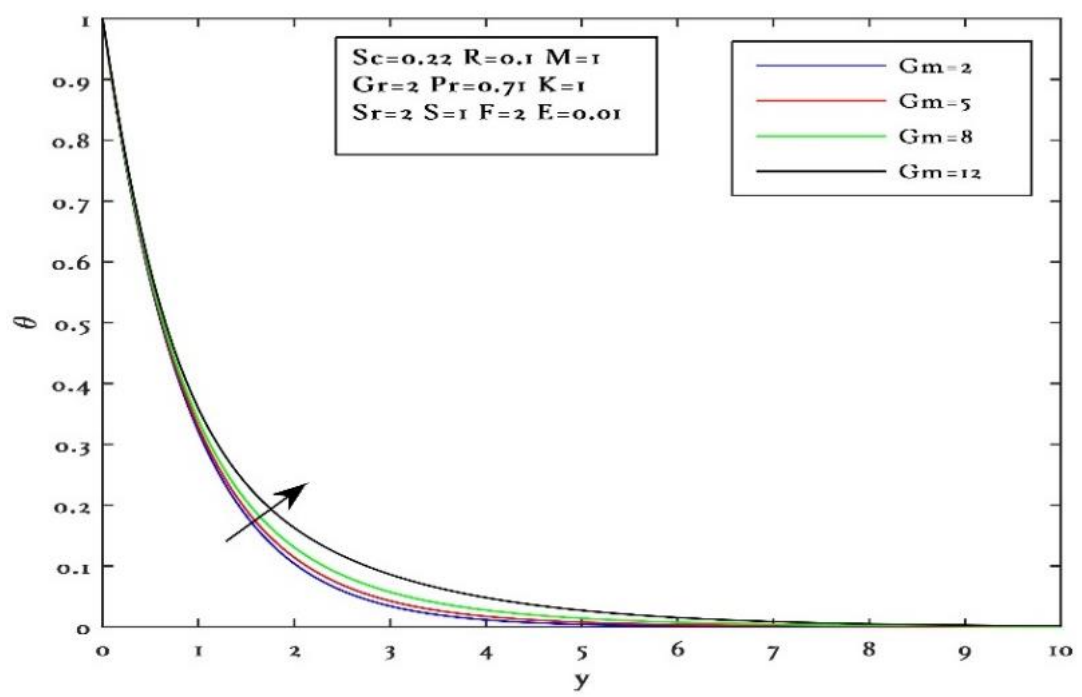

Figure 10. Temperature for different values of Gm when $\alpha=\frac{\pi}{6}$.

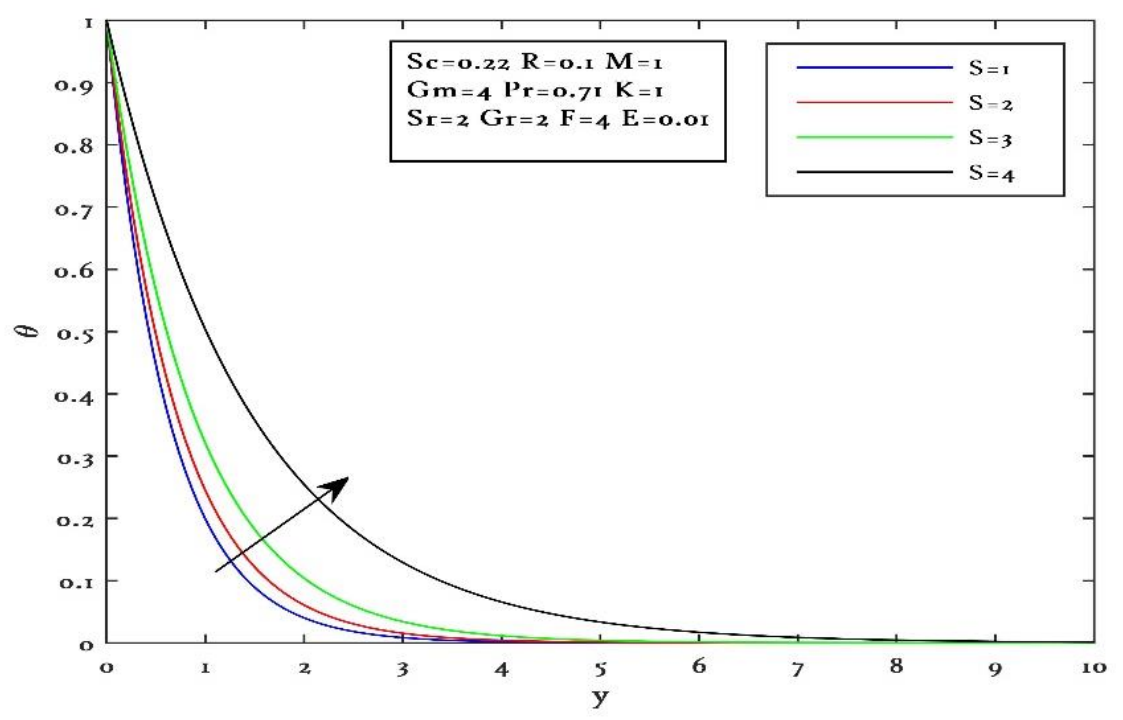

Figure 11. Temperature for different values of $\mathrm{S}$ when $\alpha=\frac{\pi}{6}$.

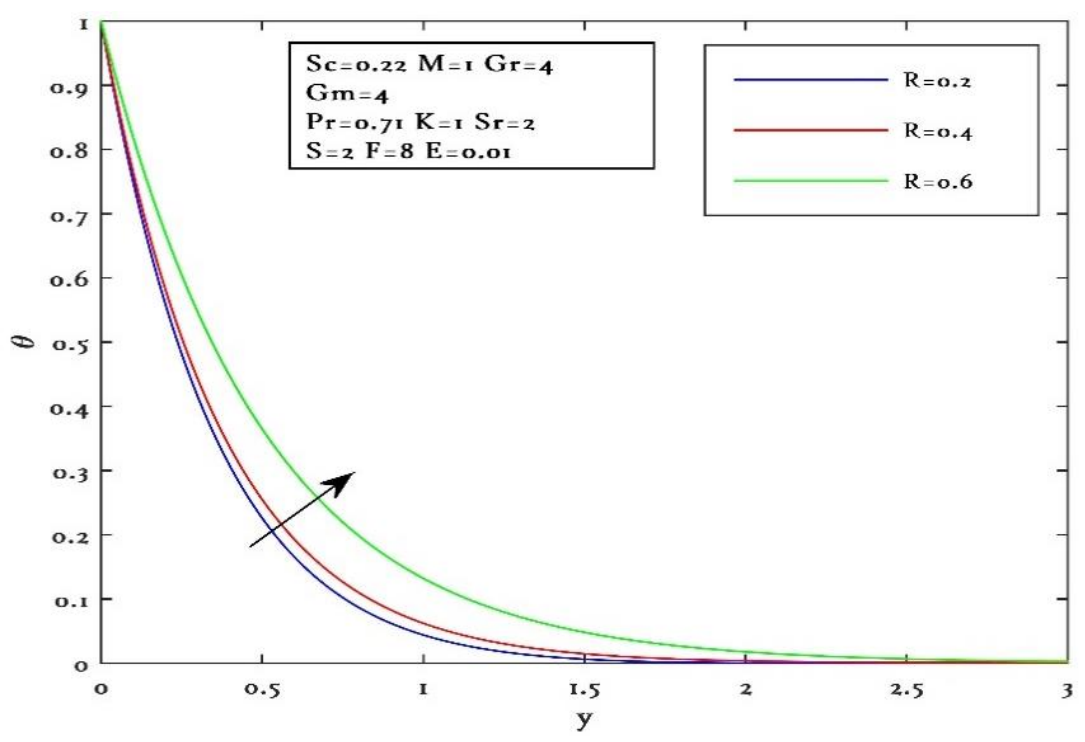

Figure 12. Temperature for different values of $\mathrm{R}$ when $\alpha=\frac{\pi}{6}$. 


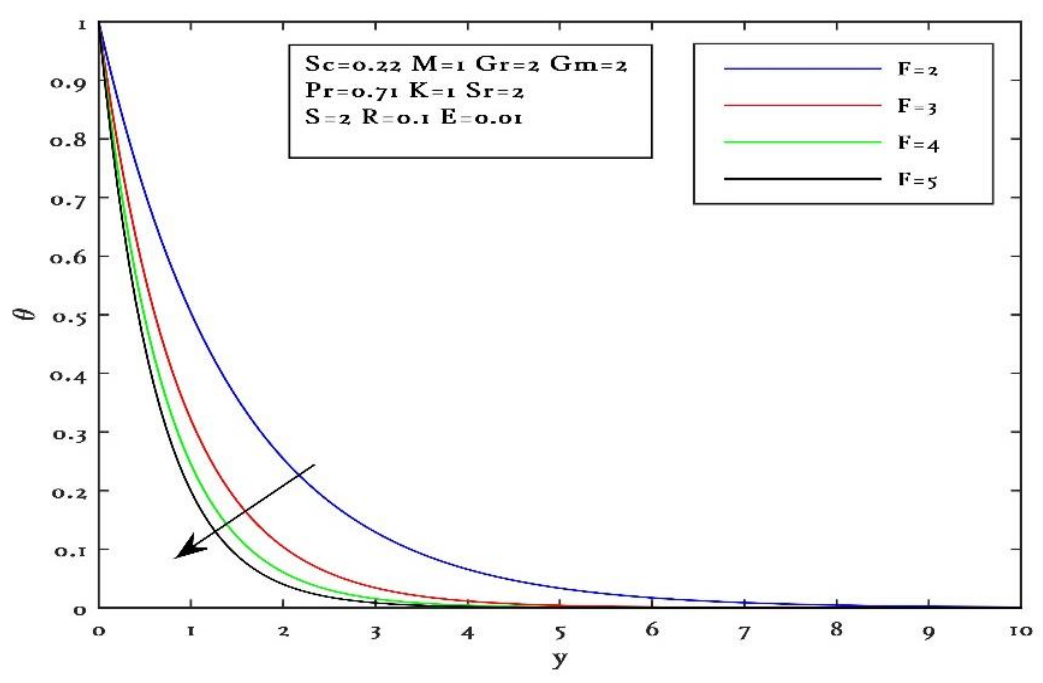

Figure 13. Temperature for different values of $\mathrm{F}$ when $\alpha=\frac{\pi}{6}$.

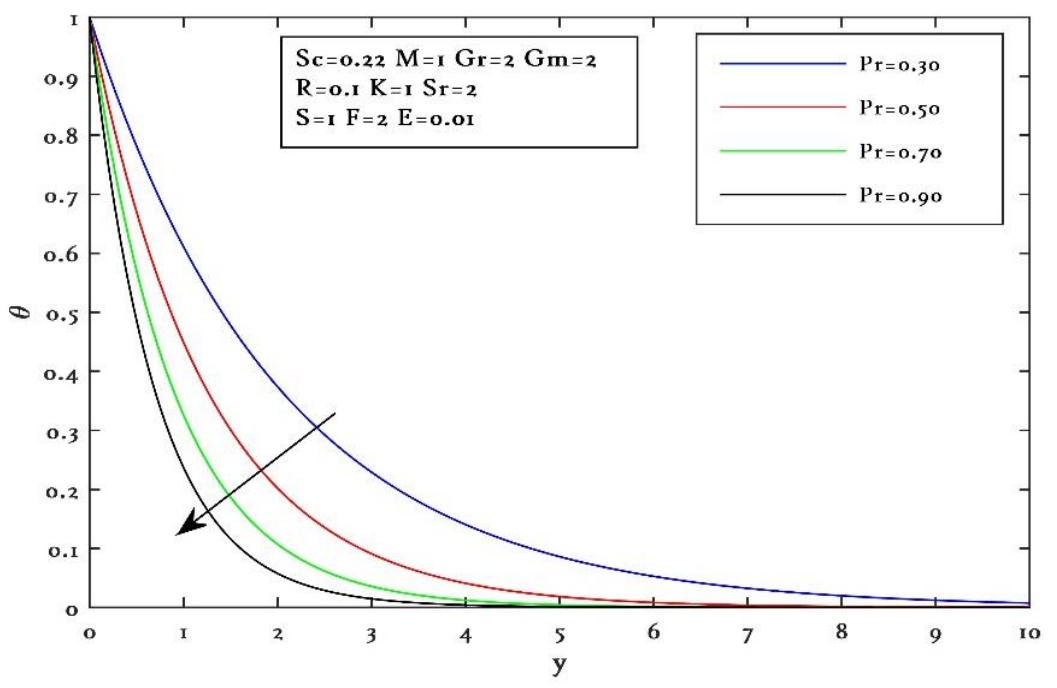

Figure 14. Temperature for different values of $\operatorname{Pr}$ when $\alpha=\frac{\pi}{6}$.

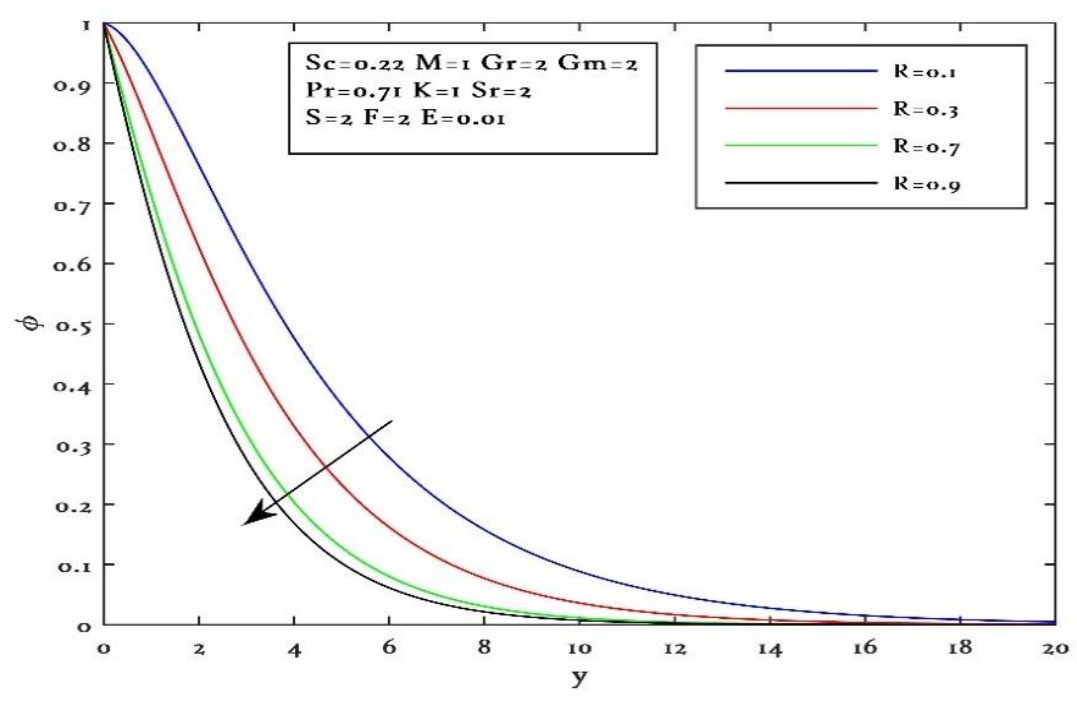

Figure 15. Concentration for different values of $\mathrm{R}$ when $\alpha=\frac{\pi}{6}$. 


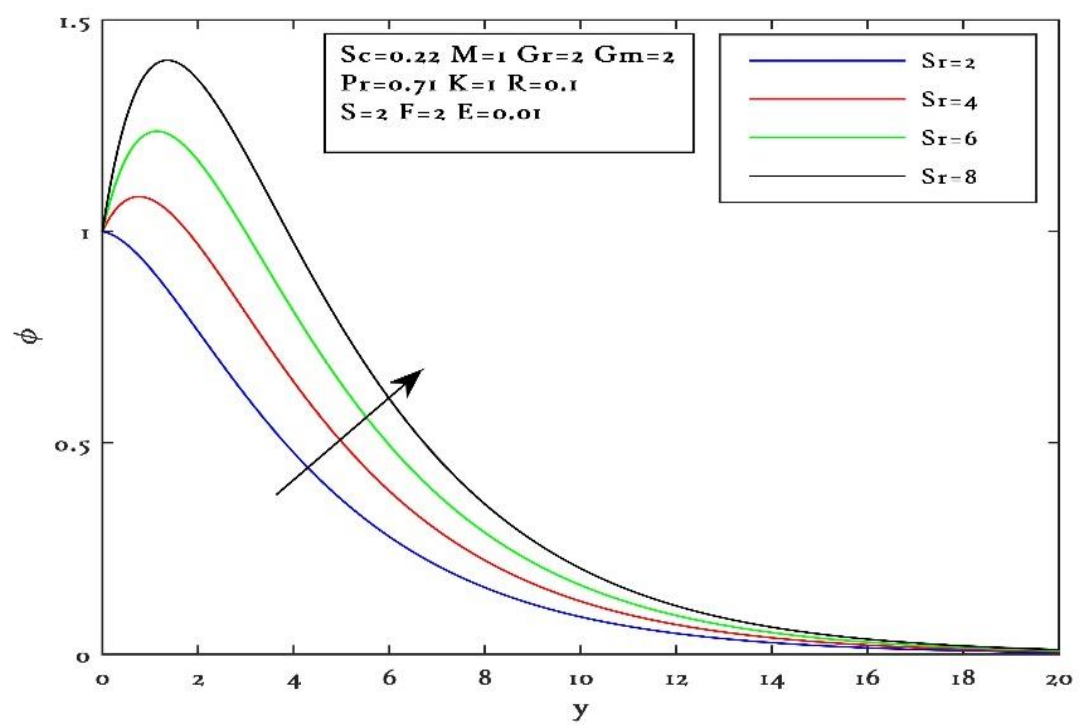

Figure 16. Concentration for different values of $\operatorname{Sr}$ when $\alpha=\frac{\pi}{6}$.

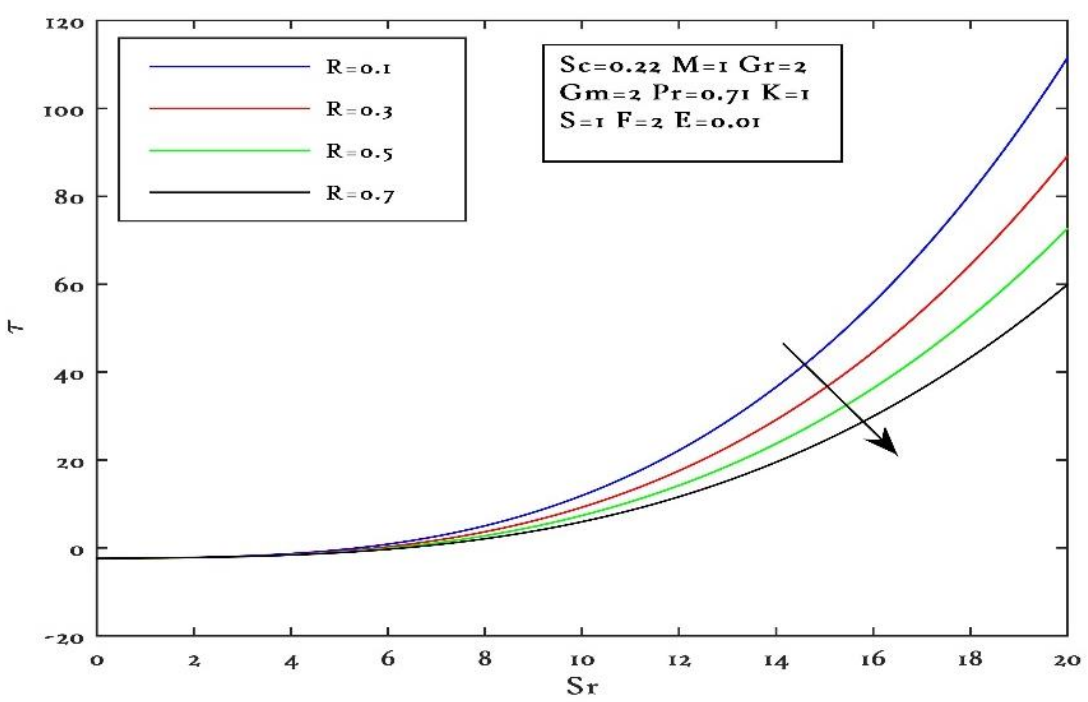

Figure 17. Skin friction for different values of $\mathrm{R}$ when $\alpha=\frac{\pi}{6}$.

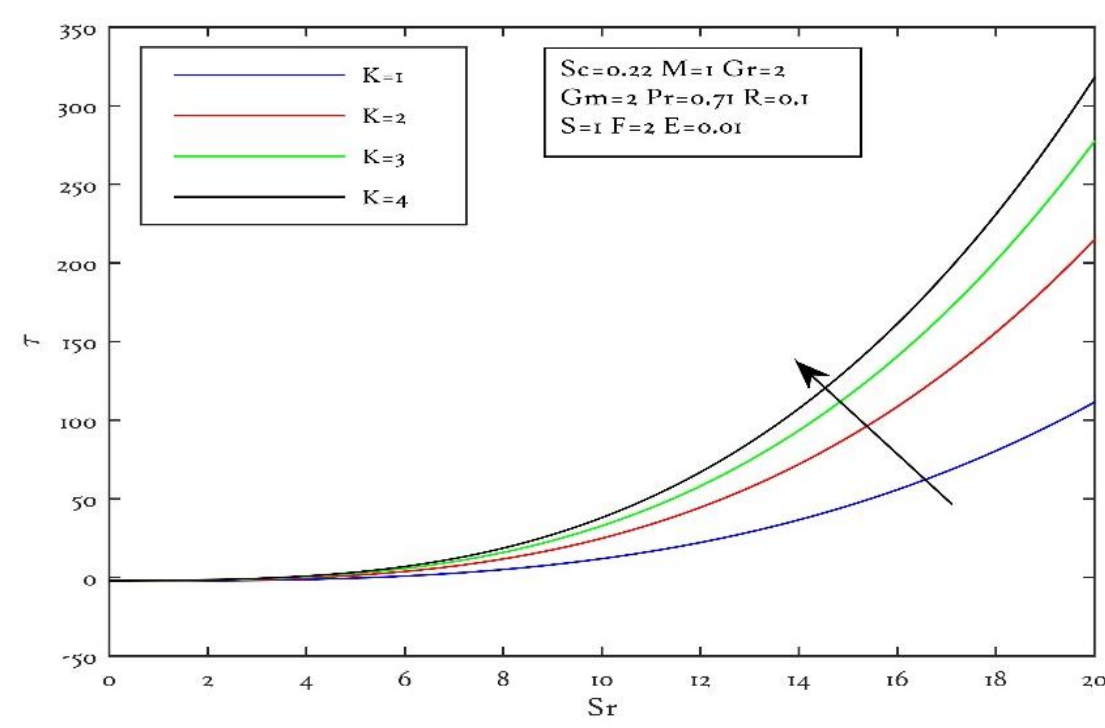

Figure 18. Skin friction for different values of $\mathrm{K}$ when $\alpha=\frac{\pi}{6}$. 


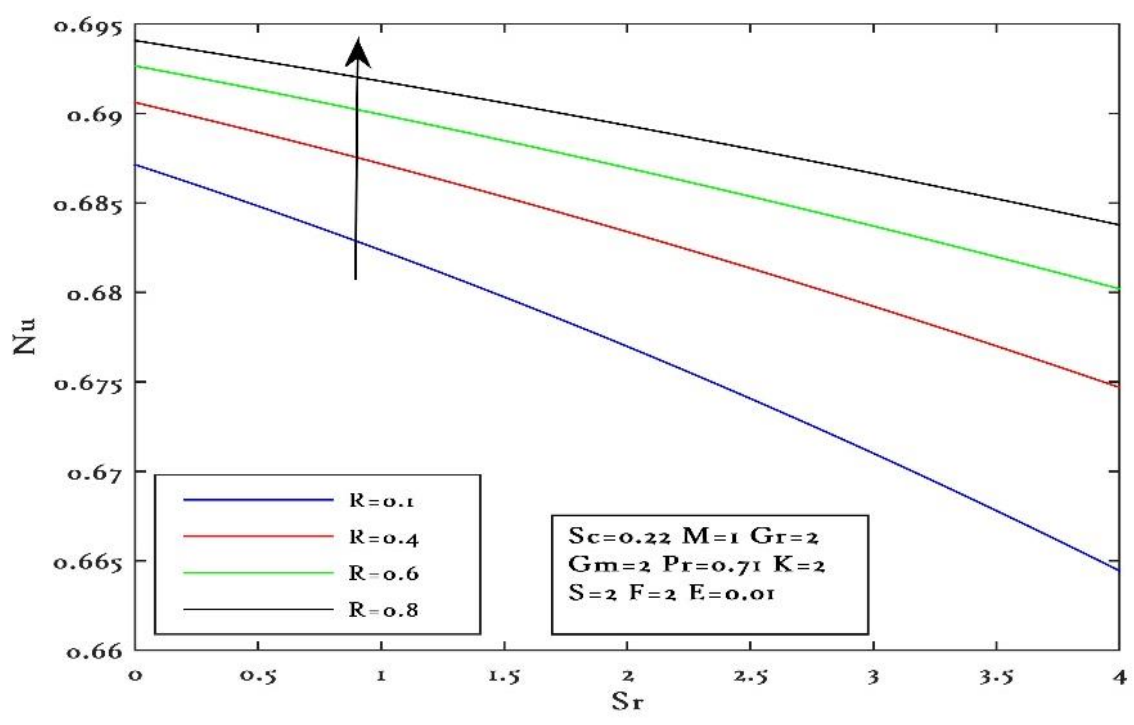

Figure 19. Nusselt number for different values of $\mathrm{R}$ when $\alpha=\frac{\pi}{6}$.

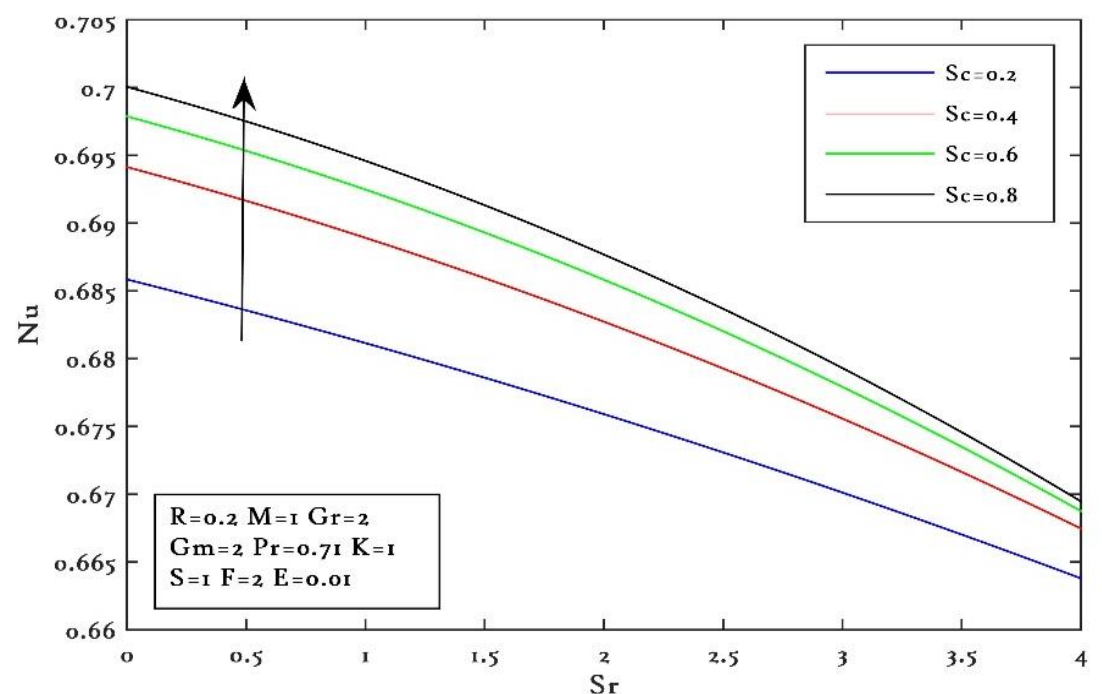

Figure 20. Nusselt number for different values of Sc when $\alpha=\frac{\pi}{6}$.

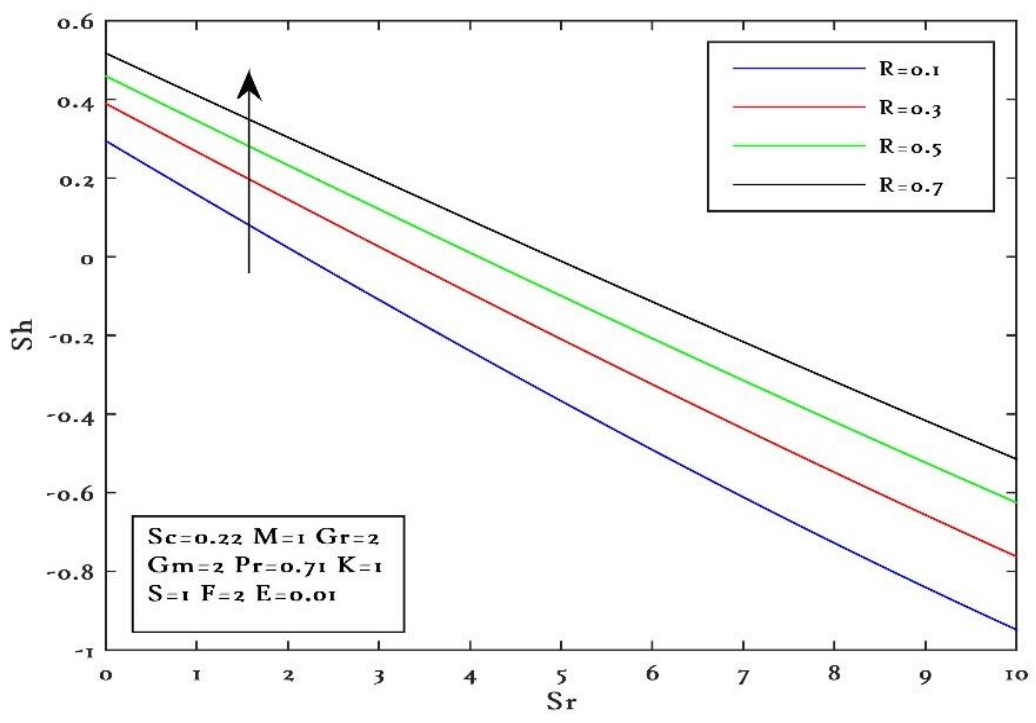

Figure 21. Sherwood number for different values of $\mathrm{R}$ when $\alpha=\frac{\pi}{6}$. 


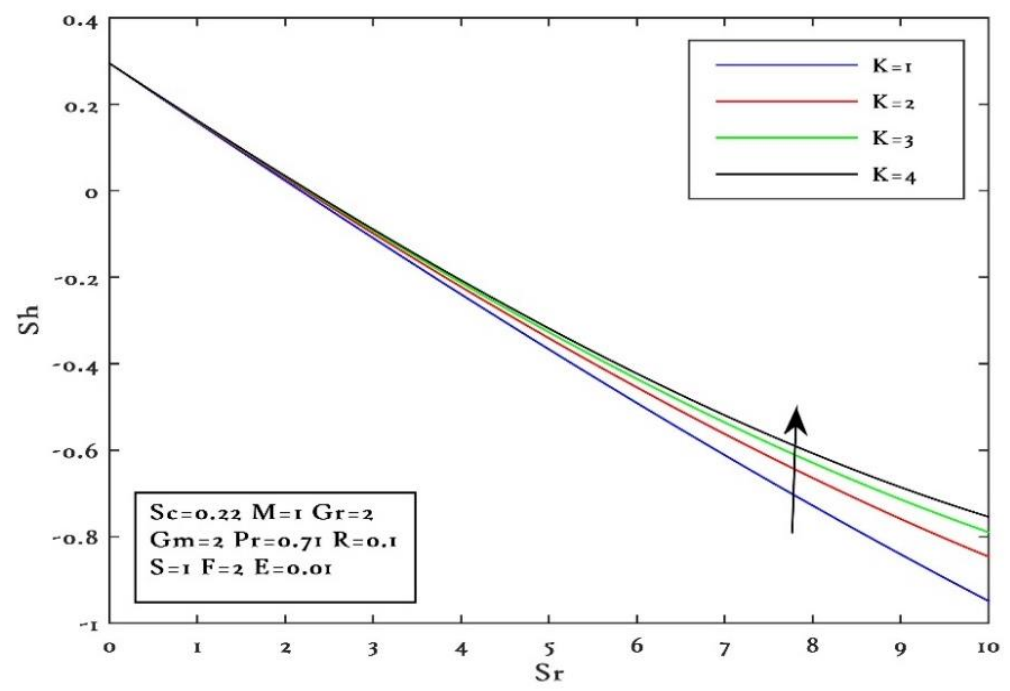

Figure 22. Sherwood number for different values of $\mathrm{K}$ when $\alpha=\frac{\pi}{6}$.

\section{Conclusions}

The velocity, concentration profiles, and skin friction fall significantly with increasing values of the chemical reaction. Temperature profiles, Nusselt number, and Sherwood number increase with the increase of chemical reaction. Heat sources increase the velocity and temperature at the plate. Velocity, skin friction, and Sherwood number increase with porosity parameter. Due to radiation parameters, velocity falls but temperature increases. A rise of the Soret number increases the velocity and concentration profiles.

\section{Appendix}

$$
\begin{aligned}
& Z=\operatorname{Pr}(F-S), \\
& Q=M+\frac{1}{K}, m_{1}=-\frac{\operatorname{Pr}+\sqrt{\operatorname{Pr}^{2}+4 Z}}{2}, m_{2}=-\frac{S c+\sqrt{S c^{2}+4 R S c}}{2}, m_{3}=-\frac{1+\sqrt{1+4 Q}}{2}, \\
& A_{1}=-\frac{m_{1}^{2} S r S c}{m_{1}^{2}+m_{1} S c-R S c}, A_{2}=1-A_{1}, A_{3}=-\frac{G r \operatorname{Cos} \alpha}{m_{1}^{2}+m_{1}-Q}, A_{4}=-\frac{A_{2} G m \operatorname{Cos} \alpha}{m_{2}^{2}-m_{2}-Q}, A_{5}=-\frac{A_{1} G m \cos \alpha}{m_{1}^{2}+m_{1}-Q}, \\
& A_{6}=-\left(A_{3}+A_{4}+A_{5}\right), A_{7}=-\frac{m_{3}{ }^{2} A_{6}{ }^{2} \operatorname{Pr}}{4 m_{3}^{2}+2 m_{3} \operatorname{Pr}-Z}, A_{8}=-\frac{m_{1}{ }^{2}\left(A_{3}+A_{5}\right)^{2} \operatorname{Pr}}{4 m_{1}{ }^{2}+2 m_{1} \operatorname{Pr}-Z}, A_{9}=-\frac{m_{2}{ }^{2} A_{4}{ }^{2} \operatorname{Pr}}{4 m_{2}{ }^{2}+2 m_{2} \operatorname{Pr}-Z}, \\
& A_{10}=-\frac{2 m_{1} m_{3} A_{6}\left(A_{3}+A_{5}\right) \operatorname{Pr}}{\left(m_{1}+m_{3}\right)^{2}+\left(m_{1}+m_{3}\right) \operatorname{Pr}-Z}, A_{11}=-\frac{2 m_{1} m_{2} A_{4}\left(A_{3}+A_{5}\right) \operatorname{Pr}}{\left(m_{1}+m_{2}\right)^{2}+\left(m_{1}+m_{2}\right) \operatorname{Pr}-Z}, \\
& A_{12}=-\frac{2 m_{2} m_{3} A_{4} A_{6} \operatorname{Pr}}{\left(m_{2}+m_{3}\right)^{2}+\left(m_{2}+m_{3}\right) \operatorname{Pr}-Z}, A_{13}=-\frac{M A_{6}^{2} \operatorname{Pr}}{4 m_{3}^{2}+2 m_{3} \operatorname{Pr}-Z}, A_{14}=-\frac{M\left(A_{3}+A_{5}\right)^{2} \operatorname{Pr}}{4 m_{1}^{2}+2 m_{1} \operatorname{Pr}-Z}, \\
& A_{15}=-\frac{M A_{4}{ }^{2} \operatorname{Pr}}{4 m_{2}{ }^{2}+2 m_{2} \operatorname{Pr}-Z}, A_{16}=-\frac{2 M A_{4} A_{6} \operatorname{Pr}}{\left(m_{2}+m_{3}\right)^{2}+\left(m_{2}+m_{3}\right) \operatorname{Pr}-Z}, A_{17}=-\frac{2 M A_{4}\left(A_{3}+A_{5}\right) \operatorname{Pr}}{\left(m_{1}+m_{2}\right)^{2}+\left(m_{1}+m_{2}\right) \operatorname{Pr}-Z}, \\
& A_{18}=-\frac{2 M A_{6}\left(A_{3}+A_{5}\right) \operatorname{Pr}}{\left(m_{1}+m_{3}\right)^{2}+\left(m_{1}+m_{3}\right) \operatorname{Pr}-Z}, A_{19}=-\left(A_{7}+A_{8}+A_{9}+A_{10}+A_{11}+A_{12}+A_{13}+A_{14}+A_{15}+A_{16}+A_{17}+A_{18}\right) \text {, } \\
& A_{20}=-\frac{m_{1}^{2} A_{19} S r S c}{m_{1}^{2}+S c m_{1}-R S c}, A_{21}=-\frac{4 m_{3}^{2}\left(A_{7}+A_{13}\right) S r S c}{4 m_{3}^{2}+2 S c m_{3}-R S c}, A_{22}=-\frac{4 m_{1}^{2}\left(A_{8}+A_{14}\right) S r S c}{4 m_{1}^{2}+2 S c m_{1}-R S c},
\end{aligned}
$$




$$
\begin{aligned}
& A_{23}=-\frac{4 m_{2}^{2}\left(A_{9}+A_{15}\right) S r S c}{4 m_{2}^{2}+2 S c m_{2}-R S c}, A_{24}=-\frac{\left(m_{1}+m_{3}\right)^{2}\left(A_{10}+A_{18}\right) S r S c}{\left(m_{1}+m_{3}\right)^{2}-S c\left(m_{1}+m_{3}\right)-R S c}, \\
& A_{25}=-\frac{\left(m_{1}+m_{2}\right)^{2}\left(A_{11}+A_{17}\right) S r S c}{\left(m_{1}+m_{2}\right)^{2}-S c\left(m_{1}+m_{2}\right)-R S c}, A_{26}=-\frac{\left(m_{2}+m_{3}\right)^{2}\left(A_{12}+A_{16}\right) S r S c}{\left(m_{2}+m_{3}\right)^{2}+S c\left(m_{2}+m_{3}\right)-R S c}, \\
& A_{27}=-\left(A_{20}+A_{21}+A_{22}+A_{23}+A_{24}+A_{25}+A_{26}\right), A_{28}=-\frac{\left(A_{19} G r+A_{20} G m\right) \cos \alpha}{m_{1}{ }^{2}+m_{1}-Q}, \\
& A_{29}=-\frac{\left\{\left(A_{7}+A_{13}\right) G r+A_{21} G m\right\} \cos \alpha}{4 m_{3}{ }^{2}+2 m_{3}-Q}, A_{30}=-\frac{\left\{\left(A_{8}+A_{14}\right) G r+A_{22} G m\right\} \cos \alpha}{4 m_{1}{ }^{2}+2 m_{1}-Q}, \\
& A_{31}=-\frac{\left\{\left(A_{9}+A_{15}\right) G r+A_{23} G m\right\} \cos \alpha}{4 m_{2}{ }^{2}+2 m_{2}-Q}, A_{32}=-\frac{\left\{\left(A_{10}+A_{18}\right) G r+A_{25} G m\right\} \cos \alpha}{\left(m_{1}+m_{3}\right)^{2}+\left(m_{1}+m_{3}\right)-Q}, \\
& A_{33}=-\frac{\left\{\left(A_{11}+A_{17}\right) G r+A_{24} G m\right\} \cos \alpha}{\left(m_{1}+m_{2}\right)^{2}+\left(m_{1}+m_{2}\right)-Q}, A_{34}=-\frac{\left\{\left(A_{12}+A_{16}\right) G r+A_{26} G m\right\} \cos \alpha}{\left(m_{2}+m_{3}\right)^{2}+\left(m_{2}+m_{3}\right)-Q}, A_{35}=-\frac{A_{27} G m \cos \alpha}{m_{2}{ }^{2}+m_{2}-Q}
\end{aligned}
$$

\section{Funding}

\section{This research article received no external funding.}

\section{Acknowledgments}

The authors would like to thank the editorial board and reviewers for their valuable comments to improve the paper's quality.

\section{Conflicts of Interest}

\section{The authors declare no conflict of interest.}

\section{References}

1. Alfven, H. Existence of electromagnetic-hydrodynamic wave. Nature 1942, 150, 405-406, https://doi.org/10.1038/150405d0.

2. Cowling, T.G. Magnetohydrodynamics, Wiley Interscience, New York, 1957.

3. Ferraro, VCA and Plumpton, C. An Introduction to Magneto-Fluid Mechanics, Geophysical journal International, 1967,12, 543-544, https://doi.org/10.1093/gji/12.5.543-a.

4. Cramer, KR and Pai, SI. Magneto Fluid Dynamics for Engineers and applied physicists, McGraw-Hill Book co., New York, 1973.

5. Singh, N.P.; Singh, A.K. MHD effects on Heat and Mass transfer in flow of viscous fluid with induced magnetic field. Ind. J. Pure Appl. Physics 2000, 38, 182-189.

6. Singh, N.P.; Singh, A.K. MHD free convection and mass transfer flow past a flat plate. Arabian Journal for Science and Engineering 2007, 32, 93-112.

7. Shateyi, S. Heat and mass transfer for natural convection MHD flow over a permeable moving vertical plate with convective boundary condition in the presence of viscous dissipation. AIP Conf. Proc. 2017, 1863, 560075, https://doi.org/10.5098/hmt.9.7.

8. Lawal, K.K.; Jibril, H.M. Impact of relative motion of a magnetic field on unsteady magnetohydrodynamic natural convection flow with a constant heat source/sink. Heat Transfer 2021, 50, 487-507, https://doi.org/10.1002/htj.21888.

9. Ramakrishna, S.B.; Thavada, S.K.; Venkatachala, G.M.; Bandaru, M. Impacts of Chemical Reaction, Diffusion-Thermo and Radiation on Unsteady Natural Convective Flow past an Inclined Vertical Plate under Aligned Magnetic Field. Biointerface research in applied chemistry 2021, 11, 13252-13267, https://doi.org/10.33263/BRIAC115.1325213267. 
10. Ilias, M.R.; Ismail, N.S.; Ab Raji, N.H.; Rawi, N.A.; Shafie, S. Unsteady aligned MHD boundary layer flow and heat transfer of a magnetic nanofluids past an inclined plate. International Journal of Mechanical Engineering and Robotics Research 2020, 9, 197-206, https://doi.org/10.18178/ijmerr.9.2.

11. Jawad, M.; Saeed, A.; Khan, A.; Alrabaiah, H; Gul, Taza; Bonyah, E.; Zubair, M. Analytical study of MHD mixed convection flow for Maxwell nanofluid with variable thermal conductivity and Soret and Dufour effects. AIP Advances 2021, 11, https://doi.org/10.1063/5.0029105.

12. Masood, S.; Farooq, M.; Ahmad, S. Description of viscous dissipation in Magnetohydrodynamic flow of nanofluid: applications of biomedical treatment. Adv Mech Eng 2020, 12, 1-13, https://doi.org/10.1177/1687814020926359.

13. Ajibade, A.O.; Umar, A.M.; Kabir, T.M. An analytical study on effects of viscous dissipation and suction/injection on a steady MHD natural convection couette flow of heat generating/absorbing fluid. Advances in Mechanical Engineering 2021, 13, https://doi.org/10.1177/16878140211015862.

14. Devi, G.L.; Niranjan, H.; Sivasankaran, S. Chemical reaction, radiation and activation energy effects on MHD buoyancy induced nanofluid flow past a vertical surface. 2021, https://doi.org/10.24200/SCI.2021.56835.4934.

15. Ganga, B.; Govindaraju, M.; Hakeem, A.A. Effects of inclined magnetic field on entropy generation in nanofluid over a stretching sheet with partial slip and non-linear thermal radiation. Iran J. Sci. Technol.- Trans. Mech. Eng 2019, 43, 707-718.

16. Dar, A.A. Effect of thermal radiation, temperature jump and inclined magnetic field on the peristaltic transport of blood flow in an asymmetric channel with variable viscosity and heat absorption/generation. Iran J. Sci. Technol.-Trans. Mech. Eng, 2020, 45, 487-501, https://doi.org/10.1007//s40997-020-00349-6.

17. Selvarasu, M.; Balamurugan, K. Effect of viscous dissipation ohmic heating and hall current on MHD free convection flow past an inclined porous plate in the presence of Dufour effect, heat source and chemical reaction. Science, technology and development, 2021, 10, 221-232.

18. Parashar, P.J.; Ahmed, N. Mass Transfer Effect on a Rotating MHD Transient Flow of Liquid Lead Through a Porous Medium in Presence of Hall and Ion Slip Current with Radiation. Mathematical Modelling of Engineering Problems, 2021, 8, 134-141, https://doi.org/10.18280/mmep.080117.

19. Ahmed, N. Soret and radiation effects on transient MHD free convection from an impulsively started infinite vertical plate. J. Heat Transf. 2012, 134, 062701, https://doi.org/10.1115/1.4005749.

20. Jena, S; Dash, G.C.; Mishra, S.R. Chemical reaction effect on MHD viscoelastic fluid flow over a vertical stretching sheet with heat source/sink. Ain Shams Eng. Journal 2018, 9, 1205-1213, https://doi.org/10.1016/j.asej.2016.06.014.

21. Gurram, D.; Balamurugan, K.S.; Raju, V.C.C.; Vedavathi, N. Effect of chemical reaction on magnetohydrodynamics casson fluid flow past an inclined surface with radiation. Skit Res. J. 2017, 7, 53-59, https://192.168.1.13:8080/xmlui/handle/123456789/705.

22. Raju, R.S. Transfer effects on an unsteady MHD mixed convective flow past a vertical plate with chemical reaction. Engineering Transaction, 2017, 65, 221-249.

23. Rashidi, M.M; Ali, M.; Rostami, B.; Rostami, P.; Xie, G.N. Heat and mass transfer for MHD viscoelastic fluid flow over a vertical stretching sheet with considering Soret and Dufour effects. Mathematical Problems in Engineering 2015, 861065, 1-12, https://doi.org/10.1155/2015/861065.

24. Venkateswarlu, M.; Reddy, G.V.R.; Lakshmi, D.V. Radiation effects on MHD boundary layer flow of liquid metal over a porous stretching surface in porous medium with heat generation. J. KSIAM 2015, 19, 83-102, https://dx.doi.org/10.12941/jksiam.201519.083.

25. Bilal, M.; Nazeer, M. Numerical analysis for the non-Newtonian flow over stratified stretching/shrinking inclined sheet with the aligned magnetic field and non-linear convection. Archive of Applied Mechanics 2020, 91, 949-964, https://doi.org/10.1007/s00419-020-01798-w.

26. Usharani, V.; Selvaraj, A.; Constance Angela, R.; Neel Armstrong, A. Impact of magnetohydrodynamics stream past an exponentially inclined vertical plate of first order chemical response with variable mass diffusion with variable mass diffusion and thermal radiation. Mathematical modelling and computational Science, 2021, 1292, 485-497.

27. Megaraju, P.; Shekar, M.N.R. Transient MHD flows through flows through an exponentially accelerated isothermal vertical plate with Hall effect and chemical reaction effect. Partial differential equations in applied Mathematics 2021, 4, https://doi.org/10.1016/j.padiff.2021.100047. 
28. Reddy, N.N.; Reddy, B.R. Chemical reaction impact on MHD natural convection flow through porous medium past an exponentially stretching sheet in presence of heat source/sink and viscous dissipation. Case studies in thermal engineering 2021, 25, 100879, https://doi.org/10.1016/j.csite.2021.100879.

29. Oyekunle, T.L.; Agunbiade, S.A. Diffusion-thermo and thermal-diffusion effects with inclined magnetic field on unsteady MHD slip flow over a permeable vertical plate. Journal of Egyptian Mathematical Society 2020, 28, 1-19, https://doi.org/10.1186/s42787-020-00110-7.

30. Sehra; Haq, SU; Shah, SIA; Nisar, KS; Jan, SU and Khan, I. Convection heat mass transfer and MHD flow over a vertical plate with chemical reaction, arbitrary shear stress and exponential heating. Scientific reports, 2021, 11, https://doi.org/10.1038/s41598-021-81615-8. 